\title{
Marketing adaptado al territorio: Place Marketing como herramienta de gestión de la imagen de destino
}

\author{
Marta PluMED LASARTE \\ Universidad de Zaragoza \\ mplumed@unizar.es
}

Recibido: 17-12-2012

Aceptado: 21-01-2013

\begin{abstract}
RESUMEN
Cuando un sujeto recibe una información acerca de un territorio, crea una imagen mental del mismo que será más positiva o más negativa en función de la información recibida y de cómo el sujeto procese dichos datos. Por ello, las Administraciones pertinentes desarrollan diferentes acciones de gestión de imagen, lo que ha dado lugar al establecimiento del concepto "marketing territorial" (place marketing), desarrollado con el objetivo de promocionar diferentes características de los territorios en función de los objetivos planteados y el público al que se dirijan. En este artículo se recopila información sobre el desarrollo, las características diferenciales, los tipos y la gestión de este tipo de estrategias de marketing territorial con el objetivo de comprender y confirmar la relevancia que la gestión de la imagen territorial ha ido adquiriendo por parte del sector público.
\end{abstract}

Palabras clave: marketing, territorio, destino, imagen. 


\title{
Marketing in territories: \\ Place Marketing as a tool for destination image management
}

\begin{abstract}
When a subject receives information about a territory, he/she creates a mental image of it which will be positive or negative depending on that information and how the subject processes those data. Thus, the different Administrations develop image management actions, establishing the concept of place marketing, developed with the objective of promoting different characteristics of the territories according to the planned goals and the target consumers. This paper compiles information about the development, the distinguishing characteristics, the types and the management of this kind of place marketing strategies with the aim of comprehending and confirming the relevance that territorial image management has been getting from the public sector.
\end{abstract}

Keywords: marketing, territory, destination, image.

Sumario: 1. Introducción. 2. Adaptación del marketing al territorio: place marketing. 3. Discusión y conclusiones.

\section{INTRODUCCIÓN}

Todo territorio, ya sea una ciudad, un país, una aldea o una isla, transmite una imagen a todos los públicos que influirá en sus opiniones sobre el mismo. Por ello, cada vez son más las Administraciones que tratan de gestionar su imagen para lograr que ésta sea siempre positiva y que transmita el mensaje deseado a sus diferentes sectores. Esta tendencia de gestión de imagen por parte de los territorios está creando un mercado de destinos cada vez más competitivo por varios motivos (Pike, 2004):

- Intangibilidad y riesgo, ya que el hecho de que el producto que un territorio "vende" sea intangible lleva a que se considere un mayor riesgo en la decisión de compra.

- Inseparabilidad y variabilidad, en cuanto a que algunos consumidores son parte del producto (residentes), además de que éste se produce y se consume al mismo tiempo. Por otro lado, la satisfacción va a depender de muchos factores, algunos de los cuales son incontrolables por parte de los responsables de la gestión de la imagen.

- El producto es perecedero, los servicios no pueden ser almacenados y guardados.

- El producto es altamente sustituible dado el alto nivel de competitividad que hay en el mercado, lo cual deja al factor imagen un papel importante. 
Otro motivo que explica la cada vez más necesaria gestión de la imagen por parte de los diferentes territorios son los cambios que se están realizando en la regulación de los sistemas de economía y finanzas con el objetivo de que los territorios atraigan más inversores, trabajadores y residentes (Avraham y Ketter, 2008). Por ello se están haciendo esfuerzos en relación a la imagen transmitida, dada su contribución a la competitividad del territorio. Este hecho, aunque sea tratado como una tendencia más bien novedosa, ya quedaba manifiesto en 1990 cuando Ashworth y Voogd explicaban que la percepción de las ciudades, definida como la imagen mental que se tiene de ellas, conforma un componente activo de su éxito o fracaso económico. Por tanto, conocer cómo las personas ven un territorio es fundamental para su desarrollo. Analizando cada segmento de mercado y diferenciando entre la percepción de los visitantes y de los que todavía no lo son, el territorio podrá descubrir si satisface las expectativas o qué es lo que más atrae a los diferentes sectores (Anholt, 2009).

Según Kotler et al. (1999), entre el 5 y el $10 \%$ de los espacios publicitarios en periódicos como el Financial Times es ocupado por este llamado marketing territorial, que para estos autores consiste en diseñar todos los aspectos de un lugar con el fin de que satisfaga las necesidades y expectativas de su público objetivo considerando todos sus componentes: su carácter, su infraestructura, sus servicios básicos, sus atractivos y sus residentes. Gold y Ward (1994), añaden que en el proceso de marketing territorial interviene activamente ese público objetivo como creadores de imágenes "deseables". Según Avraham y Ketter (2008), los estudios de marketing territorial se fundamentan en dos bases teóricas: la que conecta estas estrategias con la economía política, involucrando al Gobierno local y a la comunidad de negocios; y la que se concentra en el éxito de varias estrategias.

Tal y como definen Kavaratzis y Ashworth (2008), son varios los acontecimientos que han llevado a que los territorios lleven a cabo estrategias de marketing. Por un lado, los territorios tienen actualmente un conjunto de ventajas y desventajas con las que deben operar en diferentes mercados debido a la nueva economía global. Por otro lado se han ido produciendo una serie de cambios en el modo en que la sociedad se organiza, particularmente en la relación entre el Gobierno y los gobernados. Además, estos cambios han modificado la naturaleza de la identidad de los territorios, el modo en el que éstos son vistos y las experiencias que se viven en ellos y, por tanto, también las expectativas que las personas tienen de los territorios y de los responsables de su gestión.

Por tanto, a pesar de que durante mucho tiempo los territorios desarrollaban su promoción y comercialización sin plan ni estrategia alguna, los diferentes órganos responsables se han ido percatando de la amenaza de la competencia tanto a nivel nacional como internacional, que comenzó en el sector turístico principalmente (Lanquar, 2001). Estas nuevas exigencias del mercado han dado lugar al desarrollo del marketing territorial, cuya conceptualización, tipos y gestión son analizados en este artículo a través de una exhaustiva revisión de la literatura interdisciplinar. 


\section{ADAPTACIÓN DEL MARKETING AL TERRITORIO: PLACE MARKETING}

El fenómeno de la aplicación de los conocimientos de marketing en los procesos operacionales de los territorios no es algo nuevo. De la competitividad ya comentada entre territorios nace la necesidad de distinguirse del resto para poder así destacar la individualidad y las características diferenciadoras con el objetivo de alcanzar sus objetivos económicos, sociales y políticos, satisfaciendo al mismo tiempo las necesidades de sus consumidores actuales y potenciales. Para ello, los Gobiernos saben que deben tener clara la identidad del lugar para promocionarla en los diferentes mercados, tanto internos como externos (Kavaratzis y Ashworth, 2008). Según De Elizagarate (2008), es a finales del siglo XX y como consecuencia de la crisis económica internacional de entonces cuando se comienza a modificar la gestión tradicional del territorio y se va introduciendo el marketing por parte de la Administración, principalmente a nivel urbano.

Según Braun et al. (2010), O’Leary e Iredal (1976) fueron los primeros en identificar el marketing territorial como un nuevo campo de estudio, describiéndolo como actividades "diseñadas para crear disposiciones favorables y comportamientos hacia localizaciones geográficas". Estos autores también explican que en los años siguientes, las primeras publicaciones que se dedicaron al marketing territorial eran a nivel regional y venían de economistas, geógrafos y otros científicos sociales, pero todavía se centraban en aspectos promocionales. A principios de los 90 empezó a darse el cambio y las contribuciones se ampliaron a un marco de planificación estratégica para el marketing territorial (Ashworth y Voogd, 1990), y de los cambios estructurales que éste puede conllevar en ciudades y regiones (Berg y Braun, 1999).

\section{a) Características especiales}

Esta concepción del marketing aplicado a territorios supone tratar a éstos de una forma empresarial, contando además con diferentes herramientas de gestión que serán fundamentales para competir en el mercado y destacar sus ventajas competitivas. Sin embargo, estos procesos de marketing en ocasiones nos llevan a tratar al territorio como producto y al público objetivo como consumidores de este producto. Estas dos perspectivas nos llevan a reflexionar sobre si se debe tratar al territorio como empresa o como producto, siendo ésta una de las características que diferencian en mayor medida al marketing territorial del marketing general de otras empresas, pero no la única.

Otra característica propia de los territorios que diferencia sus procesos de marketing de los demás es la importancia que recae en las personas que lo consumen. Tal y como describen Kavaratzis y Ashworth (2008), el hecho de que la gente se identifique con ciertos lugares va más allá del propio marketing territorial, 
lo cual asimismo va más allá de la creación y promoción del imaginario como parte de la gestión del territorio. Consumir un territorio no es lo mismo que consumir un producto, ya que existe una parte emocional más importante creada desde la experiencia personal y esa unión de carácter único que se da con el territorio, en relación con conceptos como la identidad o el sense of place. Además, el marketing se sustenta en las necesidades humanas, ya sean físicas, sociales (pertenencia y afecto) o individuales (conocimiento y expresión de la personalidad) (Armstrong y Kotler, 2002), todas ellas, como vemos, íntimamente relacionadas con el territorio, ya sea desde el punto de vista de residente como desde el punto de vista de un visitante. A este respecto, De Elizagarate (2008) habla de marketing interno cuando se busca la satisfacción e identificación de los propios residentes, y de marketing externo cuando se trata de atraer y satisfacer las necesidades de los inversores, empresas, turistas, visitantes, etc.

Siguiendo con las características específicas de este tipo de marketing, podemos resumir que éste se diferencia por los siguientes aspectos (Zenker y Braun, 2011):

- Variedad de públicos objetivo, tanto internos como externos.

- Variedad en el tipo de oferta, en los diferentes "productos" que ofrece el territorio.

- No tiene ánimo de lucro directo, sino que el objetivo es maximizar el desarrollo económico y social del lugar.

- Involucración política.

- Diferentes percepciones en cuanto a la imagen.

b) Tipos de marketing territorial

Dada la variedad de públicos objetivo a los que se dirige un mismo territorio, las acciones y estrategias de marketing serán diferentes para cada uno de ellos ya que su visión del lugar y los objetivos que buscan en relación a él son muy distintos (Paz, 2005; De Elizagarate, 2008). Sin embargo, aunque las acciones de marketing territorial que se desarrollan desde la Administración son distintas según el consumidor al que se dirija, bien es cierto que éstas suelen agruparse de una forma más global, destacando de una manera más singular el marketing turístico.

Dentro del marketing territorial, el turístico es quizá el que mayor peso tiene en cuanto a que hay territorios que se centran mayoritariamente en él o que incluso lo ven como el único objetivo de sus acciones de marketing territorial. Tal y como explican Kavaratzis y Ashworth (2008), es en el sector turístico donde se percibe más efectividad por parte del marketing territorial, quizá por las características del turismo como proveedor de servicios que hace más sencillo el traspaso de conocimiento a esta industria.

De hecho, son más las publicaciones y los estudios relacionados con el marketing turístico que con el marketing territorial en general, quizá también porque los resultados del primero son más fáciles de medir y las acciones están 
dirigidas a un público que va a dejar un beneficio económico en el territorio, mientras que el marketing dirigido a residentes y trabajadores actuales y potenciales no representa una inversión a tan corto plazo. A este respecto, acentuándose todavía más actualmente dada la difícil situación económica en la que se encuentran numerosos territorios, es frecuente ver cómo las estrategias y acciones de marketing que se desarrollan son aquellas que pueden tener un beneficio económico en el menor plazo posible, dejando de lado las igualmente necesarias inversiones sociales.

Sin embargo, dejando a un lado la situación económica actual que dificulta cualquier tipo de análisis coyuntural, sí es cierto que echando la vista atrás se podría decir que la tendencia del marketing territorial está pasando de ser meramente de promoción turística a una comunicación más rica y global. Por este motivo vamos a centrarnos en estos dos grandes grupos: marketing de destino turístico y marketing territorial a nivel global.

El marketing de destino turístico puede ser descrito como el proceso de gestión mediante el cual las organizaciones y/o empresas turísticas identifican a sus turistas actuales y potenciales así como sus deseos, necesidades, motivaciones, preferencias y no preferencias, tanto a nivel local como regional, nacional e internacional, formulando y adaptando sus productos turísticos con el objetivo de lograr la satisfacción óptima del turista mientras se cumplen sus objetivos (Wahab et al., 1976, citados en Pike, 2004). Según explica Lanquar (2001), ya desde las primeras definiciones de marketing turístico se tenían en cuenta las preocupaciones de los destinos turísticos, incluso en mayor medida que de las empresas turísticas. Sin embargo, en su libro "Marketing turístico", Lanquar deja claro que el marketing turístico se refiere tanto a empresas como a organismos de marketing de destinos, con sus características concretas en cada caso. De cualquier manera, el marketing turístico se encarga de comprender las características especiales tanto del mercado turístico como de su oferta y demanda, lo cual ayuda al desarrollo de estrategias de marketing adecuadas por parte de los diferentes destinos. Un ejemplo lo encontramos en el análisis de Serra (2005), que clasifica los condicionantes del comportamiento del turista de la siguiente manera: 
Tabla 1. Condicionantes del comportamiento del turista

\begin{tabular}{|l|l|}
\hline CONDICIONANTES INTERNOS & CONDICIONANTES EXTERNOS \\
\hline Motivaciones para viajar & Entorno económico, político, legal, cultural... \\
\hline Percepción & Grupos de referencia \\
\hline Experiencia y aprendizaje & Familia \\
\hline Actitudes & Determinantes situacionales \\
\hline Características personales & Clase social \\
\hline
\end{tabular}

Fuente: Elaboración propia en base a Serra (2005)

Kotler et al. (2003), por su parte, clasifican las características personales que afectan a comportamiento del consumidor en cuatro grupos:

Tabla 2. Factores influyentes en el comportamiento del consumidor

\begin{tabular}{|l|l|l|l|}
\hline $\begin{array}{l}\text { FACTORES } \\
\text { CULTURALES }\end{array}$ & $\begin{array}{l}\text { FACTORES } \\
\text { SOCIALES }\end{array}$ & $\begin{array}{l}\text { FACTORES } \\
\text { PERSONALES }\end{array}$ & $\begin{array}{l}\text { FACTORES } \\
\text { PSICOLÓGICOS }\end{array}$ \\
\hline Cultura & $\begin{array}{l}\text { Grupos de referencia, } \\
\text { pertenencia... }\end{array}$ & Edad y momento vital & Motivación \\
\hline \multirow{2}{*}{ Subcultura } & Familia & Ocupación & Percepción \\
\hline Clase social & Rol y status & $\begin{array}{l}\text { Circunstancias } \\
\text { económicas }\end{array}$ & Aprendizaje \\
\cline { 3 - 4 } & & Estilo de vida & Creencias y actitudes \\
\cline { 3 - 3 } & & $\begin{array}{l}\text { Personalidad y concepto } \\
\text { de uno mismo }\end{array}$ & \\
\hline
\end{tabular}

Fuente: Elaboración propia en base a Kotler et al. (2003)

Por otro lado, Serra (2005) resume también las características especiales que tiene un turista en su comportamiento de compra frente a un consumidor normal:

- Elevado componente emocional

- Elevado nivel de implicación y compromiso

- Elevado nivel de incertidumbre debido a la intangibilidad del producto 
- Elevado nivel de influencia de otras personas

- Antelación en la decisión

- Elevado nivel de búsqueda de información

Estas características son las que hacen que el proceso de compra en turismo sea más complejo que un proceso de compra de un producto común, algo que el marketing debe comprender para hacer frente a esas especialidades y comprender las necesidades del cliente. Si bien es cierto que muchas de estas características no pueden ser controladas desde el marketing, han de ser consideradas para así tratar de influir en el comportamiento del consumidor y, por lo tanto, en su toma de decisiones. Por tanto, en relación con los conceptos ya analizados de imagen, vemos que una adecuada estrategia de marketing puede ayudar a cambiar la percepción que el turista potencial tiene de un destino, aumentando las posibilidades de elección y mejorando su posicionamiento. Sin embargo, el marketing turístico no sólo debe comprender las características del consumidor, sino que también debe analizar los componentes del destino como producto, los cuales se pueden resumir en (Middleton, 1994; citado por Serra, 2005):

- Los atractivos del destino

- Las instalaciones y servicios del destino

- La accesibilidad al destino

- La imagen del destino

- El precio global que supone para el consumidor

Otro aspecto que analiza el marketing turístico es el ciclo de vida del producto (CVP), el cual aparece prácticamente en cualquier manual de marketing. Su aplicabilidad al destino turístico fue realizada por primera vez por Butler (1980, citado en Serra, 2005), cuya hipótesis parte de que el ciclo de vida de un destino es similar al CVP general, por lo que sigue las siguientes fases: exploración, implicación, desarrollo, consolidación y estancamiento, que puede dar lugar a rejuvenecimiento/estabilización o declive.

Por otro lado, el marketing territorial global abarca todo tipo de acciones, ya que tiene en cuenta todos los consumidores posibles, incluidos los turistas y visitantes, los cuales son el único público objetivo en el tipo anterior. Cuando un territorio decide llevar a cabo una estrategia de marketing a nivel global el planteamiento es diferente y por lo tanto las acciones desarrolladas también, motivo por el cual se ha tomado como un tipo de marketing concreto, cuyas estrategias, tal y como explica De Elizagarate (2008), tratan de conseguir que el territorio sea un lugar atractivo para vivir, trabajar, invertir y visitar. En el marketing territorial, el público objetivo al que se dirigen las acciones está formado no sólo por aquellos consumidores potenciales a los que se pretende atraer, sino también por todos los consumidores actuales, siendo éstos aquellos que tengan una relación con el lugar, sea del tipo que sea. Tal y como explican Kavaratzis y Ashworth (2008) el marketing puede desarrollar un incremento del atractivo del lugar de cara no sólo a visitantes sino a muchos otros públicos, comenzando por los propios residentes. También 
analizan el hecho de que un territorio no es sólo un destino turístico y centrar todos los esfuerzos en atraer visitantes no sólo limita peligrosamente la efectividad del marketing sino que puede dañar el propio carácter del lugar, ya que enfocar el territorio sólo al turismo probablemente no encaje con las aspiraciones de los residentes. Hay que tener en cuenta que los residentes no son solamente uno de los principales públicos objetivo sino que son los que más influyen en los otros. Por ese motivo sus necesidades y deseos deben ser parte integral de los objetivos del territorio y deben formar parte, por lo tanto, del desarrollo de todas las etapas de la estrategia de marketing.

Uno de los avances más interesantes en marketing de lugares en los últimos años según Avraham y Ketter (2008) es el uso de la visión del lugar como marco para el proceso de marketing. Esta estrategia consiste en averiguar cuál es la visión que se quiere dar del lugar, para lo cual es interesante conocer las diferentes visiones de los residentes respecto a los distintos factores del lugar, abarcando así los objetivos que se desean conseguir. Con relación a la perspectiva de los residentes, De Elizagarate (2008) destaca que la investigación académica ha ido teniendo en cuenta de forma creciente la calidad de vida como variable generadora de competitividad entre territorios, lo cual no sólo es importante de cara a la opinión local, sino que hace al lugar más atractivo para consumidores potenciales. Por otro lado, a pesar de que los residentes son una parte esencial del marketing territorial a nivel global, hay otros públicos que no son menos importantes. En el siguiente gráfico (Figura 1) de Kotler y Gretner (2002), vemos que los cuatro públicos objetivo más importantes son (a) los visitantes, (b) los residentes y trabajadores, (c) los negocios e industria y (d) los mercados de exportación. Las acciones que se desarrollen para atraer o mantener a cada uno de ellos serán diferentes en función de los objetivos que se persigan con cada uno.

Figura 1. Niveles del marketing territorial

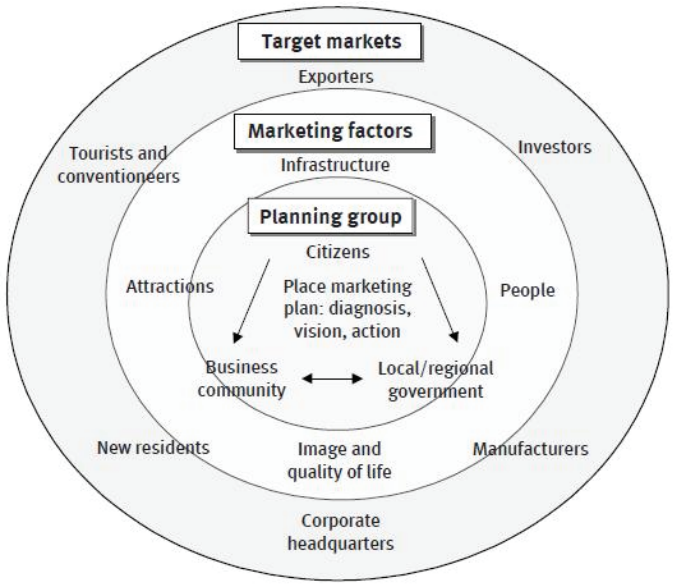

Fuente: Kotler y Gertner, 2002 
En esta figura, target markets se refiere a los públicos objetivo ya citados, los segmentos de clientes a los cuales se les transmiten diferentes mensajes; marketing factors son los atractivos e infraestructuras del lugar, así como su población, su imagen y su calidad de vida; y planning groups son los grupos responsables del planteamiento y control del marketing territorial.

Los mismos autores describen además que los objetivos de los diferentes consumidores van a determinar su proceso de toma de decisiones a la hora de elegir uno u otro destino, lo que ellos llaman los factores de atracción, que dividen en:

- Factores duros (hard factors), los que son más o menos medibles objetivamente:

- Estabilidad económica

- Productividad

- Costes

- Concepto de propiedad

- Servicios y redes de apoyo local

- Infraestructura de comunicación

- Localización estratégica

- Esquemas y programas de incentivos

- Factores blandos (soft factors), los que no son fácilmente medibles y representan características más subjetivas del lugar:

- Nicho del mercado

- Calidad de vida

- Competencias profesionales

- Cultura

- Personal

- Gestión

- Flexibilidad y dinamismo

- Profesionalismo en contacto con el mercado

- Mundo empresarial

Según Pride (2008), citado por Morgan et al. (2011), diferenciarse a través de hard factors como la infraestructura, la economía, la accesibilidad o las características financieras del lugar es cada vez más complicado. Sin embargo, soft factors como el ambiente, la amabilidad de la población local, el entretenimiento y los servicios de ocio, así como las tradiciones y la cultura del destino están ganando más importancia a la hora de atraer tanto inversionistas como a turistas.

c) Gestión del marketing territorial

Tal y como se ha ido describiendo, las políticas públicas responsables deben saber gestionar el marketing de su territorio como una herramienta de estrategia de desarrollo que contribuya a determinar su identidad y el posicionamiento percibido 
por el público objetivo potencial, al mismo que tiempo que se trata de incrementar el sentimiento de arraigo en los propios residentes (Azevedo, 2005). Los órganos responsables de un territorio deben tomar una serie de decisiones en relación con los objetivos, las políticas y las estrategias tanto a corto como a medio y largo plazo en relación con el proceso de marketing del lugar (Lanquar, 2001). Esta gestión se lleva a cabo a partir de diferentes figuras y acciones que se han ido desarrollando con este fin, de las cuales destacamos las Destination Management Organizations (DMO).

A pesar de que las DMO han jugado desde hace tiempo un papel importante en el desarrollo territorial en todo el mundo -principalmente a nivel turístico dado que consideran el territorio como destino-, éste es un campo de estudio que sólo últimamente ha llamado la atención de investigadores, según la revisión literaria realizada por Pike (2004), la cual revela una falta de atención investigadora hacia muchos de los aspectos de las DMO a pesar de que fue a partir de 1990 cuando aparecieron textos en relación a la planificación, el marketing y la gestión territorial.

Las DMO se crearon con el objetivo de que existiera una entidad encargada de "vender" un destino, ayudando no sólo a aumentar su competitividad a largo plazo, sino actuando también como representantes de la comunidad local, el entorno natural y de los servicios turísticos, por lo que recae sobre ellas una gran obligación social. Resumiendo, los objetivos clave de las DMO para alcanzar esa competitividad se pueden agrupar en (Pike, 2004):

- Mejorar la imagen del destino

- Aumentar el aprovechamiento de la industria

- Reducir la estacionalidad

- Asegurar la financiación a largo plazo

Por otro lado, dado el papel que llevan a cabo las DMO en el sector turístico, la OMT desarrolló un estudio ${ }^{1}$ en 2004 sobre estas organizaciones a nivel mundial, el cual muestra una perspectiva de cómo se desarrolla la promoción turística a nivel internacional, clasificando estas organizaciones en tres bloques: nacionales, regionales y locales o de ciudad. De este estudio destacamos varios datos de interés, que se han agrupado según el tipo de información que aportan:

- En cuanto al modelo organizativo,

- La gran mayoría (el 88\%) de las DMO son un departamento del Gobierno central, o bien un organismo dependiente del mismo.

\footnotetext{
1 "Survey of Destination Management Organisations Report" (OMT, 2004)
} 
- A nivel regional, la mayoría de estas organizaciones dependen del Gobierno autonómico o provincial, y en ellas interviene el sector privado de manera más frecuente que a nivel nacional.

- El 39\% de las DMO de ciudad dependen del Gobierno regional, provincial o local y el porcentaje de organizaciones públicoprivadas es el más alto de todos los niveles $(33 \%)$.

- Si atendemos al presupuesto con el que cuentan,

- Casi dos de cada tres DMO nacionales tienen una inversión anual de más de 15 millones de dólares.

- Cerca el $60 \%$ de las DMO regionales y de ciudad tienen un presupuesto que supera el millón y medio de dólares.

- En relación a la fuente de ingresos,

- Las DMO nacionales obtienen más de un $60 \%$ de su presupuesto del Gobierno central, siendo la publicidad la segunda fuente más importante.

- El 29\% de las DMO regionales reciben sus fondos del Gobierno central, y el $48 \%$ obtiene casi la mitad de su presupuesto del Gobierno autonómico o local.

- Según sus características, las DMO de ciudad obtienen un $40 \%$ de su presupuesto mediante su actividad o publicidad, $\mathrm{y}$ prácticamente todas reciben fondos del Gobierno regional, provincial o local, siendo para casi la mitad la fuente principal de sus ingresos.

- En cuanto a la importancia de las actividades que realizan, las más valoradas por las DMO a todos los niveles son:

- Búsqueda de mercados.

- Publicación de tendencias turísticas internacionales.

Como vemos, estas organizaciones reciben una alta consideración por parte de la Administración pública a todos los niveles, lo cual demuestra que son órganos relevantes a la hora de la gestión territorial, principalmente a nivel turístico.

\section{DISCUSIÓN Y CONCLUSIONES}

Actualmente vivimos en una sociedad caracterizada por una creciente globalización, facilidad de acceso a todo tipo de información, ciclos económicos cambiantes, etc. Por otro lado, gracias a la proliferación del low cost y a la cantidad de oferta existente, viajar ha dejado de ser un privilegio para unos pocos 
convirtiéndose en una necesidad para muchos. Toda esta situación ha generado en los diferentes territorios una necesidad de atraer visitantes, nuevos residentes, inversiones, empresas... para así poder seguir desarrollándose y permanecer o posicionarse en un mercado de destinos que cada vez se antoja más competitivo.

Esta creciente competencia ha llevado a las Administraciones públicas responsables a prestar atención a la imagen transmitida, puesto que de ella dependerá el nivel de atracción que cada lugar tendrá en los diferentes sectores. Por tanto, cada territorio promocionará y gestionará su imagen en función de los objetivos planteados y del público al que se quiera dirigir. De esta forma, existen lugares centrados en la promoción turística, otros que enfocan sus acciones en el desarrollo empresarial, algunos quieren vender productos propios del lugar y los hay, cada vez más, que promocionan el territorio en todos los sectores para su desarrollo global. Si bien es cierto que muchas economías dependen del turismo, otros factores pueden ser igual de importantes, como estimular la inversión y ayudas, motivar la llegada de nuevos trabajadores, aumentar el negocio internacional o facilitar el proceso de integración en organizaciones políticas y comerciales como la Unión Europea o la OMT, entre otros muchos intereses. Son estos motivos los que animan a un territorio a pensar en la imagen global que presenta al resto del mundo, y no centrarse únicamente en la imagen turística que puede resultar irrelevante, no ayudar o ser incluso dañina en otros aspectos, tal y como indica Anholt en Morgan et al. (2002). Por ello los territorios deben plantearse muchos objetivos al mismo tiempo: atraer empresas nuevas (nacionales y extranjeras), retener su base industrial, desarrollar nuevos "productos" para los turistas y trabajadores visitantes (Rainisto, 2003), etc.

Por tanto, para conseguir esos objetivos, los territorios han de transmitir en todo momento una imagen positiva, para lo cual han ido implementando estrategias de gestión de imagen que ha llevado a que hoy en día se hable ya de marketing territorial o place marketing.

En el momento en el que un sujeto recibe una información acerca de algo, crea una imagen mental de ese objeto que será más positiva o más negativa en función de la información recibida y de cómo el sujeto procese dichos datos. De la misma manera ocurre con los territorios y, por ello, las Administraciones pertinentes tratan de que éstos transmitan una buena imagen con el objetivo de que los diferentes colectivos tengan una opinión positiva, ya que una imagen negativa puede perjudicar su desarrollo (De Elizagarate, 2008).

Sin embargo, la imagen territorial tiene características y matices especiales que hacen de su gestión un proceso más complejo. Por un lado, la imagen será diferente según el punto de vista desde el que se analice, de tal forma que un lugar puede tener, por ejemplo, buena imagen como destino vacacional y mala imagen como lugar de trabajo o viceversa, por lo que el control de la imagen global requerirá acciones específicas para cada percepción. Por otro lado, el mensaje a transmitir será diferente en función del público al que se dirija y de los objetivos marcados, 
por lo que es importante que ese capital imagen pueda ser utilizado como herramienta estratégica de posicionamiento en los diferentes mercados y en la mente de los consumidores. Otra de las particularidades de la imagen territorial reside en su relación con la identidad del lugar y en la importancia que ésta tiene a su vez para la imagen global. Por ello, si se considera a la población local únicamente como meros consumidores se corre el riesgo de omitir los aspectos intrínsecos y multidimensionales existentes entre el sujeto y el espacio en el que vive.

En definitiva, la gestión de la imagen por parte de los territorios se antoja fundamental para un correcto desarrollo de los mismos, tanto desde el punto de vista promocional como social, ya que es esencial que la imagen transmitida sea fiel a la identidad del territorio y a la percepción de sus habitantes. Por todo ello, la Administración Pública ha de prestar atención a estas estrategias si pretende posicionar sus diferentes territorios en los mercados objetivos de una forma competitiva.

\section{BIBLIOGRAFÍA}

ANHOLT, S. (2009). Handbook on tourism destinations branding. Madrid: World Tourism Organization (WTO).

ARMSTRONG, K. P. G. y KOTLER, P. (2002). Fundamentos de mercadotecnia. México: Editorial Prentice Hall.

ASHWORTH, G.J. y VOOGD, H. (1990). Selling the city: marketing approaches in public sector urban planning. Belhaven Press.

AVRAHAM, E. y KETTER, E. (2008). Media strategies for marketing places in crisis: Improving the image of cities, countries, and tourist destinations. Routledge.

AZEVEDO, A. (2005). "Cities as brands: The role of communication strategy in place marketing context". Libro de actas $4^{\circ}$ SOPCOM, 4, 1777-1786.

BERG, L. y BRAUN, E. (1999). Urban Competitiveness, Marketing and the Need for Organising Capacity. Urban Studies, 36, 987-999.

BRAUN, E., KAVARATZIS, M. y ZENKER, S. (2010). "My city-my brand: the role of residents in place branding". Fiftieth European Regional Science Association Congress, Joenkoeping, Suecia.

DE ELIZAGARATE, V. (2008). Marketing de ciudades. Estrategias para el desarrollo de ciudades atractivas y competitivas en un mundo global. Madrid: Ediciones Pirámide.

GOLD, J.R. y WARD, S. V. (1994). Place promotion: the use of publicity and marketing to sell towns and regions. John Wiley \& Sons Ltd. 
KAVARATZIS, M. y ASHWORTH, G. (2008). Place marketing: how did we get here and where are we going? Journal of Place Management and Development, 1 (2), 150-165.

KOTLER, P. y GRETNER, D. (2002). Theoretical papers. Country as brand, product, and beyond: A place marketing and brand management perspective. Special Issue Brand Management, 9 (4-5), 249-261.

KOTLER, P.; ASPLUND, C.; REIN, I. y HEIDER, D. (1999). Marketing places Europe: Attracting investments, industries, residents and visitors to European cities, communities, regions and nations. Financial Times Prentice-Hall, Harlow.

KOTLER, P.; BOWEN, J.; MAKENS, J. C.; MORENO, R. R. y PAZ, M. D. R. (2003). Marketing para turismo. McGraw-Hill.

LANQUAR, R. (2001). Marketing turístico. Ariel Turismo.

MORGAN, N.; PRITCHARD, A. y PRIDE, R. (2002). Destination branding: creating the unique destination proposition. Oxford: Butterworth-Heinemann.

MORGAN, N.; PRITCHARD, A. y PRIDE, R. (Eds.). (2011). Destination brands: Managing place reputation. Routledge.

ORGANIZACIÓN MUNDIAL DE TURISMO (2004). Survey of Destination Management Organisations Report. Madrid: OMT

PAZ, S. (2005). Administración estratégica de marca territorial. Como construir y administrar la marca de ciudades y regiones. Revista da Administração, 45, 119.

PIKE, S. (2004). Destination marketing organisations. Routledge.

RAINISTO, S. K. (2003). Success factors of place marketing: A study of place marketing practices in Northern Europe and the United States. Tesis doctoral no publicada, Universidad de Helsinki (Finlandia).

SERRA, A. (2005). Marketing turístico. Madrid: Ediciones Pirámide, ESIC

ZENKER, S. y BRAUN, E. (2011). "Place Brand Management. A Critical Perspective on Dealing with Complex Place Brands". Simposio "11 City Design", 2011 